\title{
Characterizing a lubricant additive for 1,3,4-tri-(2-octyldodecyl) cyclopentane: Computational study and experimental verification
}

\author{
Jingyan NIAN ${ }^{1}$, Yifan SI ${ }^{1,2}$, Zhiguang GUO ${ }^{1,2, *}$, Ping GAO ${ }^{1, *}$, Weimin LIU ${ }^{1}$ \\ ${ }^{1}$ State Key Laboratory of Solid Lubrication, Lanzhou Institute of Chemical Physics, Chinese Academy of Sciences, Lanzhou 730000, China \\ ${ }^{2}$ Hubei Collaborative Innovation Centre for Advanced Organic Chemical Materials and Ministry of Education Key Laboratory for the \\ Green Preparation and Application of Functional Materials, Hubei University, Wuhan 430062, China
}

Received: 27 June 2016 / Revised: 16 August 2016 / Accepted: 22 August 2016

(C) The author(s) 2016. This article is published with open access at Springerlink.com

\begin{abstract}
In order to increase the life of spacecraft, it is important to improve the comprehensive lubrication performance. Multiple alkylated cyclopentane (MAC) lubricants are presently gaining wide acceptance for actual space applications; adding extreme pressure additive is a strategy to improve lubrication performance. In this study, taking 1,3,4-tri-(2-octyldodecyl) cyclopentane as base oil, tricresol phosphate (traditional additive) and tri-(2-octyldodecyl) phosphate (developmental additive) have been screened computationally for compatibility, shear film forming and energy dissipation. Theoretical results indicate that (a) tricresol phosphate additive is not suited for addition to 1,3,4-tri-(2-octyldodecyl) cyclopentane lubricant due to limited compatibility; (b) tri-(2-octyldodecyl) phosphate is an excellent lubricant additive due to its perfect compatibility, ease of forming a shear film on the surface of friction pairs, higher strength, and low energy dissipation; and (c) lubrication occurs through the solid-liquid composite lubrication mechanism. These theoretical results were confirmed experimentally.
\end{abstract}

Keywords: 1,3,4-tri-(2-octyldodecyl) cyclopentane; lubricant additive; computational screening; lubrication mechanism; experimental verification

\section{Introduction}

Development of space technology has led to an increase in the number of sensitive and active components. Meanwhile, the indispensability of spacecraft has necessitated an increase in their design life [1]. Therefore, it is necessary to improve the comprehensive lubrication performance of the lubricant.

Multiple alkylated cyclopentane (MAC) lubricants are presently gaining wide acceptance for space applications because of their chemical inertness, beneficial viscosity properties, low volatility [2], and low friction coefficients [3-6]. They are synthesized by

* Corresponding author: Zhiguang GUO; Ping GAO.

E-mail: zguo@licp.cas.cn (GUO); gaoping@licp.cas.cn (GAO) reacting cyclopentadiene with alcohols in the presence of a strong base. The reaction products are then hydrogenated to produce the final products, which are a mixture of di-, tri-, tetra-, or penta-alkylated cyclopentanes [7]. In order to satisfy the application requirement under special working conditions and environments, extreme pressure additives are added as a strategy to further improve lubrication performance [5, 8-10]. Traditional simulated space environment experiments have proven that additives such as tricresol phosphate [11,12] and lead naphthenate [13, 14] can significantly improve the wear resistance and other extreme pressure properties of MACs. Lead additives are toxic, so they will not be considered here.

In this study, 1,3,4-tri-(2-octyldodecyl) cyclopentane is taken as the base oil (denoted as L) and a suitable 
lubricant additive is selected utilizing a computational protocol. Two different phosphate additives-tricresol phosphate (traditional additive) and tri-(2-octyldodecyl) phosphate (guessed additive based on the similarity of structures)-are taken as research objects. The structures of the base oil and additives are shown in Fig. 1.

\section{Computational details}

In this study, several elements are carefully explored to research lubrication performance. These are: (1) compatibility of base oil with additive, (2) strength of lubricated thin film on friction pairs, and (3) energy dissipation.

\subsection{Compatibility of the additive with oil}

The compatibility of the base oil with the additive was calculated using the Blends module $[15,16]$, which combines a modified Flory-Huggins model [17] and molecular simulation techniques. The general expression for the energy of mixing of a binary system is shown by Eq. (1) [17],

$$
\frac{\Delta G}{R T}=\frac{\phi_{a}}{n_{a}} \ln \phi_{a}+\frac{\phi_{b}}{n_{b}} \ln \phi_{s}+\chi \phi_{a} \phi_{b}
$$

In the above equation, the free energy of mixing (per mole) is indicated by $\Delta G, \phi_{a}$ and $\phi_{b}$ correspond to the volume fractions of components $a$ and $b$. $n_{a}$ and $n_{b}$ are the degrees of polymerization of components $a$ and $b$. The first two terms represent the combinatorial entropy, and the last term is the free energy contribution due to the interaction. As per standard convention, $T$ is the absolute temperature and $R$ is the gas constant; $\chi$ herein can be defined through Eq. (2),

$$
\chi=\frac{E_{\text {mix }}}{R T}
$$

$E_{\text {mix }}$ is the free energy difference between the mixed and pure states, computed by Eq. (3),

$$
E_{\text {mix }}=\frac{1}{2} Z\left(E_{a b}+E_{b a}-E_{b b}-E_{a a}\right)
$$

$\mathrm{Z}$ is the coordination number and it is the intuitional index of compatibility. The larger the value of $Z$, the better compatibility there is. $E_{a b}$ and $E_{b a}$ are the binding energy between $a$ and $b$. For molecules, the binding energy is an ensemble average over molecular configurations, and similar distributions of $E_{a b}, E_{b b}$ and $E_{a a}$ combinations indicate an excellent compatibility.

\subsection{Interaction between lubricated thin film and friction pairs}

The strength of shear thin film on friction pairs can be evaluated from the following.

(1) Concentration profile of atoms nearby the friction pairs. The higher concentration of atoms near the friction pairs indicates the formation of a lubricant film. Herein, the concentration profile is obtained from the confined shear simulation, which is a non-equilibrium molecular dynamic method. The simulations are carried out by Forcite module in Material Studio version 6.0, Accelrys Software Inc. Iron $(\mathrm{Fe})$ is taken as the material for the friction pairs since it is the main component of friction pairs for

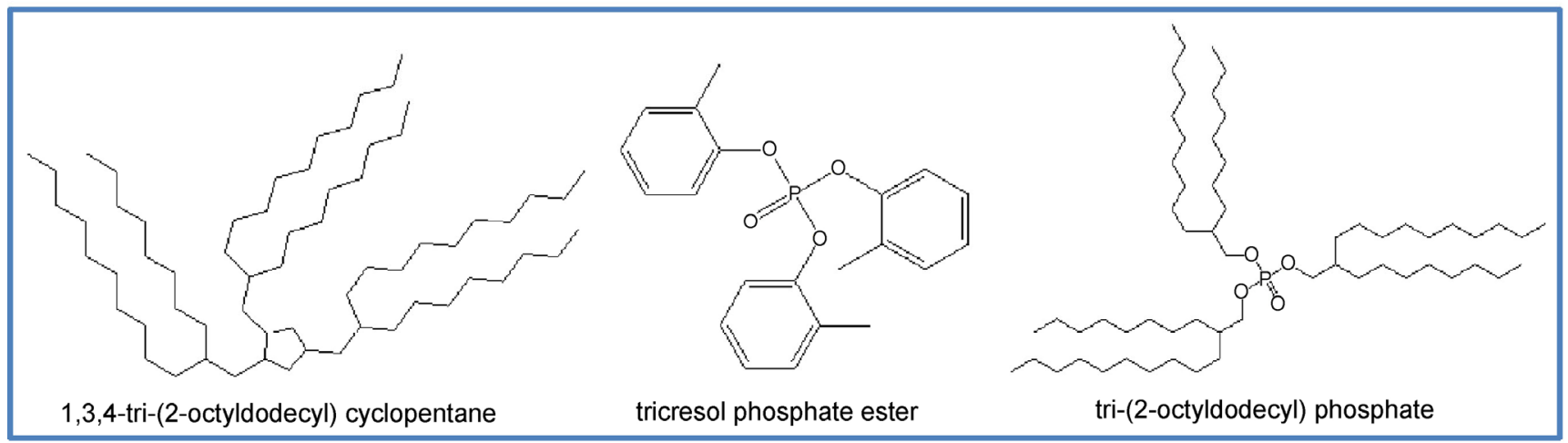

Fig. 1 The molecular structures of 1,3,4-tri-(2-octyldodecyl) cyclopentane, tricresol phosphate and tri-(2-octyldodecyl) phosphate. 
most friction test experiments. Before carrying out confined shear simulation, two amorphous cells were constructed and were filled with 1,3,4-tri-(2octyldodecyl) cyclopentane and tri-(2-octyldodecyl) phosphate, respectively. The densities of 1,3,4-tri-(2octyldodecyl) cyclopentane and tri-(2-octyldodecyl) phosphate ester are $0.85 \mathrm{~g} / \mathrm{cm}^{3}$ and $1.10 \mathrm{~g} / \mathrm{cm}^{3}$, respectively. A short energy minimization (to optimize the cell) was initially performed on both amorphous cells, followed by an annealing cycle calculation. The initial temperature was $300 \mathrm{~K}$, the mid-cycle temperature was $500 \mathrm{~K}$, and Nose thermostating algorithm was used. The annealed results are shown in Fig. 2.

The Dreiding force field [18] has been adopted for simulation calculations. The relative sliding velocity of the friction pairs is $0.2 \AA / p s$, the time step is $1 \mathrm{fs}$, and the total simulation time is $20 \mathrm{ps}$. The initial temperature of the friction pairs is $300 \mathrm{~K}$.

(2) Binding energy between lubricant molecules and friction pairs. Binding energy between lubricant molecules and friction pairs can be used to evaluate the strength of the lubricant film. A quench dynamic calculation was carried out to search for the minimum energy configuration of the base oil or additive molecule on friction. Fe $(001)(14 \times 14 \times 2)$ supercells were taken as friction pairs and the binding energy was calculated by Eq. (4),

$$
E_{\mathrm{b}}=E_{\min }-E_{\text {molecular }}-E_{\mathrm{Fe}}
$$

$E_{\mathrm{b}}$ is the binding energy of the base oil or additive with $\mathrm{Fe}(001), E_{\min }$ is the energy of minimum energy

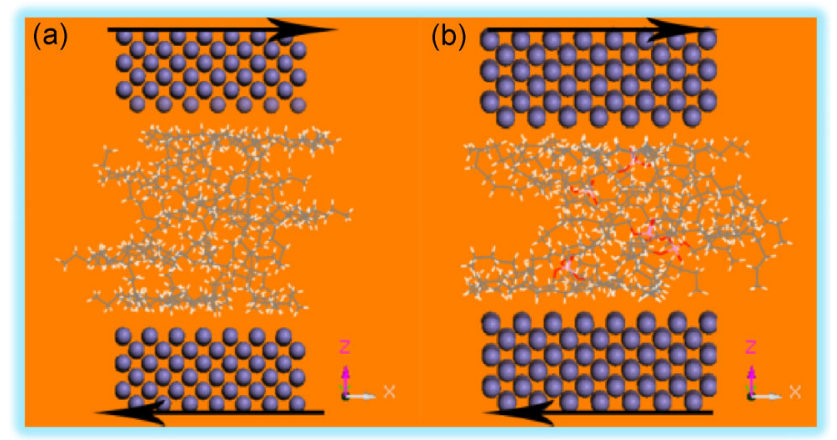

Fig. 2 The models adopted for confined shear simulation. Blue spheres indicate the friction pairs ( $\mathrm{Fe}$ atoms) and the arrows indicate the sliding direction. The middle layer indicates the lubricant: (a) 1,3,4-tri-(2-octyldodecyl) cyclopentane, and (b) tri-(2-octyldodecyl) phosphate. molecule configuration, $E_{\text {molecular }}$ represents the energy of free molecular of base oil or additive, and $E_{\mathrm{Fe}}$ is the energy of Fe (001).

\subsection{Energy dissipation}

Energy dissipation can be evaluated by shear viscosity and local heating caused by internal friction among molecules. Shear viscosity and local heating will also be obtained by confined shear simulation results from the Forcite module. In the present context, lower local heating and shear viscosity indicates the better shear slip between interlayers, and thus leads to lower energy dissipation. The shear viscosity $(\eta)$ was obtained from the numerical integration of the stress autocorrelation function (SAF), and the relation between shear viscosity and SAF can be expressed by Eq. (5) [19],

$$
\eta=\frac{V}{k T} \int_{0}^{\infty}<\sigma_{\alpha \beta}(t) \sigma_{\alpha \beta}(0)>\mathrm{d} t
$$

$\sigma_{\alpha \beta}$ denotes the three equivalent off-diagonal elements of the stress tensor, $k$ is the Boltzmann constant, $T$ is already defined earlier, and $V$ is the shear velocity.

\section{Results and discussion}

\subsection{Computational simulation results}

Compatibility calculation results are presented first. Figures 3(a) and 3(b) show that the coordination numbers for tri-(2-octyldodecyl) phosphate (denoted as $A_{1}$ ) are 6; the distribution of binding energy of tri(2-octyldodecyl) phosphate with base oil is completely identical. Meanwhile, the coordination numbers of tricresol phosphate (denoted as $\mathrm{A}_{2}$ ) with base oil are 3 , and there is some difference between $\mathrm{A}_{1}$ and $\mathrm{A}_{2}$ in the distribution of binding energy combination. Therefore, we can conclude that tricresol phosphate is not a good additive for base oil, and carrier will be needed to disperse it in base oil. However, tri-(2octyldodecyl) phosphate has an excellent compatibility with base oil.

The relative concentration of atoms $A_{1}$ is much higher than $\mathrm{L}$ in the region adjacent to the friction pairs (Fig. 4). Thus, we can conclude that $\mathrm{A}_{1}$ preferentially 

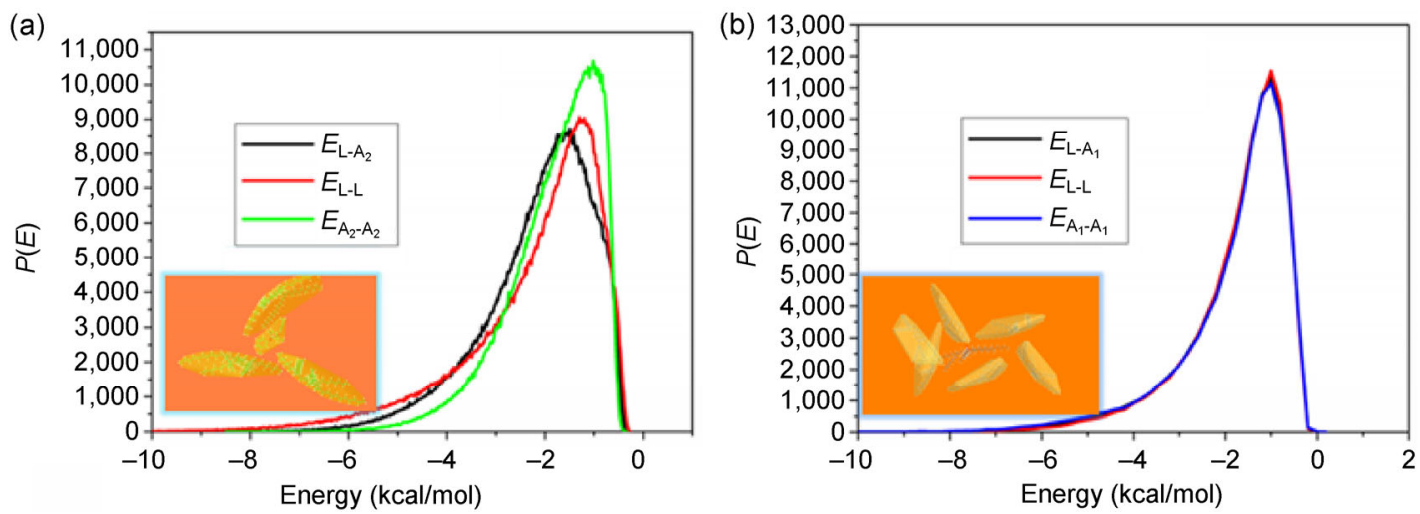

Fig. 3 Coordination numbers and binding energy distribution curves for 1,3,4-tri-(2-octyldodecyl) cyclopentane with (a) tricresol phosphate ester and (b) tri-(2-octyldodecyl) phosphate.

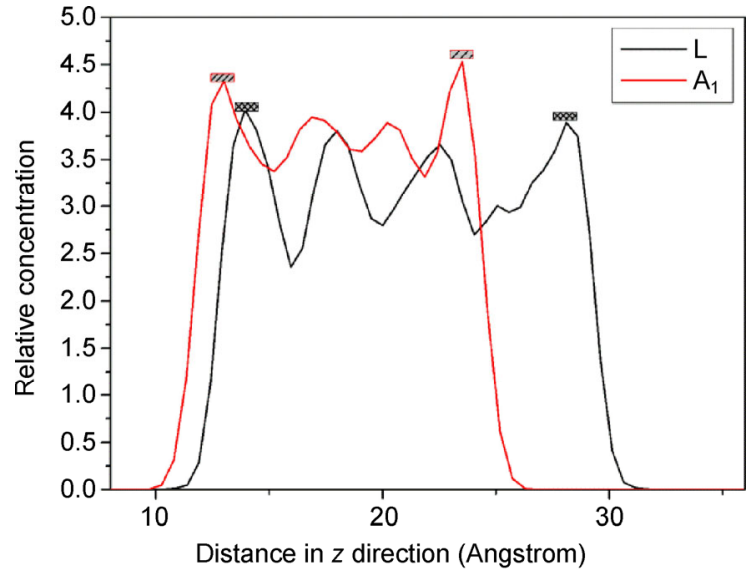

Fig. 4 The relative concentration profiles of atoms for tri-(2octyldodecyl) phosphate and 1,3,4-tri-(2-octyldodecyl) cyclopentane in the $\mathrm{z}$-direction. Rectangles indicate the locations near the friction pairs.

forms a thin film on the surface of friction pairs compared with L.

The two quench dynamic search results (Figs. 5(a) and $5(b))$ indicate that, at the minimum energy configuration of $A_{1}$, the molecule of $A_{1}$ lies completely on $\mathrm{Fe}(001)$, and the corresponding binding energy is $-511.96(\mathrm{kcal} / \mathrm{mol})$. In contrast, only a fraction of the chain of L lies on Fe (001) and the binding energy between $\mathrm{L}$ and $\mathrm{Fe}(001)$ is $-177.66(\mathrm{kcal} / \mathrm{mol})$. Therefore, the minimum energy configuration and the higher binding energy of $A_{1}$ with the surface of friction pairs demonstrate that $A_{1}$ has the higher film strength.

Figure 6 shows that $A_{1}$ has relatively low local heating during the confined shear course compared with L. The lower energy dissipation for $\mathrm{A}_{1}$ indicates a better lubrication performance when compared with $\mathrm{L}$.
Analysis of confined shear simulation results produces shear viscosities of 6.67 and 13.39 centipoise for $A_{1}$ and $L$, respectively. This indicates that $A_{1}$ has a better interlay slip corresponding to lower dissipation, and this is also the main reason for the lower local heating during friction for $\mathrm{A}_{1}$.

Based on the theoretical results, it can be concluded that tricresol phosphate is not a good additive for the base oil, and a carrier will be needed to disperse it in the base oil. Tri-(2-octyldodecyl) phosphate has excellent compatibility with base oil, strong film forming capability, and low energy dissipation, suggesting that it is an excellent lubricant additive. The difference of film forming capability between base oil and tri-(2-octyldodecyl) phosphate demonstrated that the lubrication mechanism is solid-liquid composite lubrication. Tri-(2-octyldodecyl) phosphate creates a solid-like thin film on the surface of the friction pairs, and 1,3,4-tri-(2-octyldodecyl) cyclopentane acts as the liquid component. Therefore, we can infer that lubrication performance will be improved substantially when compared the pure base oil.

\subsection{Experiment certification results}

In order to verify the conclusions of our calculations, tri-(2-octyldodecyl) phosphate was synthesized as per the literature [20]. phosphorus oxychloride (Shanghai Chemical Reagent Co., Ltd.) and synthesized 2-octyl1-dodecanol are the initial reagents. 2-octyl-1-dodecanol was synthesized according to the previously reported method [21]. The base oil tris-(2-octyldodecyl) cyclopentane was also synthesized following the same 


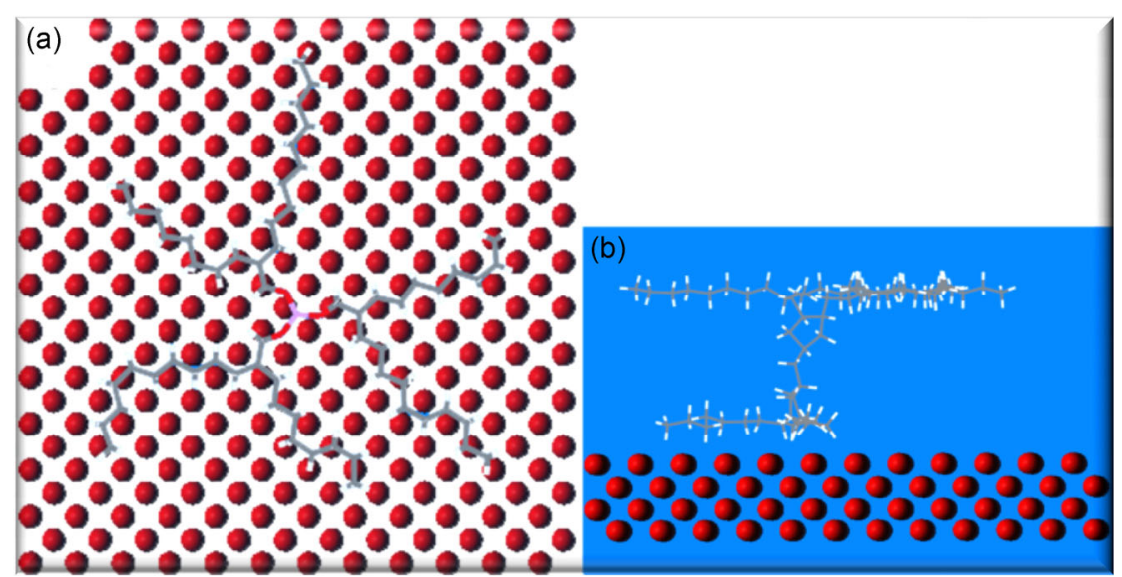

Fig. 5 The minimum energy molecular configurations on friction pairs for (a) tri-(2-octyldodecyl) phosphate molecule and (b) 1,3,4-tri(2-octyldodecyl) cyclopentane molecule.

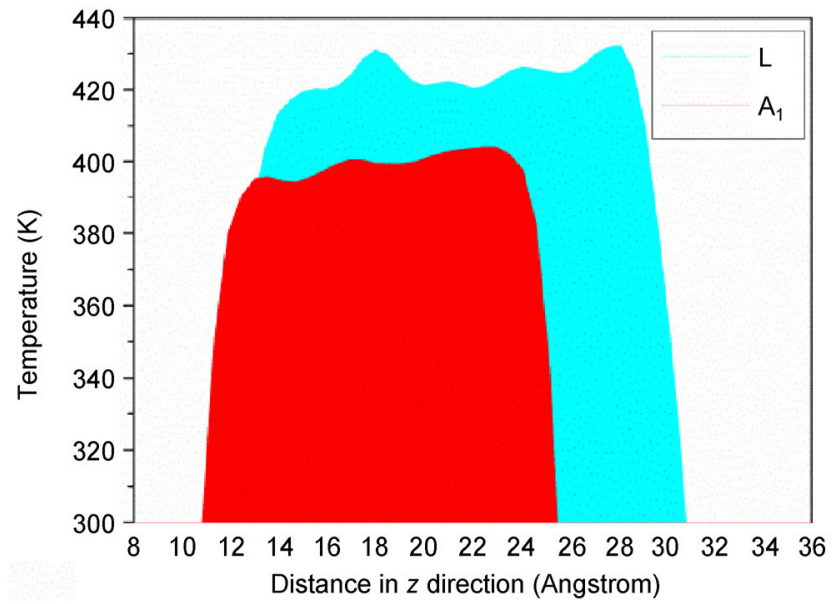

Fig. 6 Local heating caused by friction for tri-(2-octyldodecyl) phosphate and 1,3,4-tri-(2-octyldodecyl) cyclopentane.

procedure reported previously [22]. The chemical structure of the tris-(2-octyldodecyl) phosphate was characterized by IR (Nicolet iS10, Thermo Fisher Scientific) and NMR (Bruker, AVANCE III HD $400 \mathrm{MHz}$ ) spectroscopy (See Appendix). The friction tests were performed on a ball-on-disk wear tester under high vacuum conditions $\left(3 \times 10^{-4} \mathrm{~Pa}\right)$. The sliding contact was brought by pushing a $9 \mathrm{Cr} 18$ steel ball ( $3 \mathrm{~mm}$ in diameter) on a rotating $9 \mathrm{Cr} 18$ steel disk $(\Phi$ $24 \mathrm{~mm} \times 7.9 \mathrm{~mm}$ ) with a load of $3 \mathrm{~N}$. The sliding speed was $300 \mathrm{rpm}$ and the test time was $1 \mathrm{~h}$. The result of the experiment is shown in Fig. 7.

The coefficient of friction decreases substantially by up to $30 \%$ due to the addition of tris-(2-octyldodecyl) phosphate, which can powerfully verify the conclusion of our theoretical calculations. Thus we can conclude

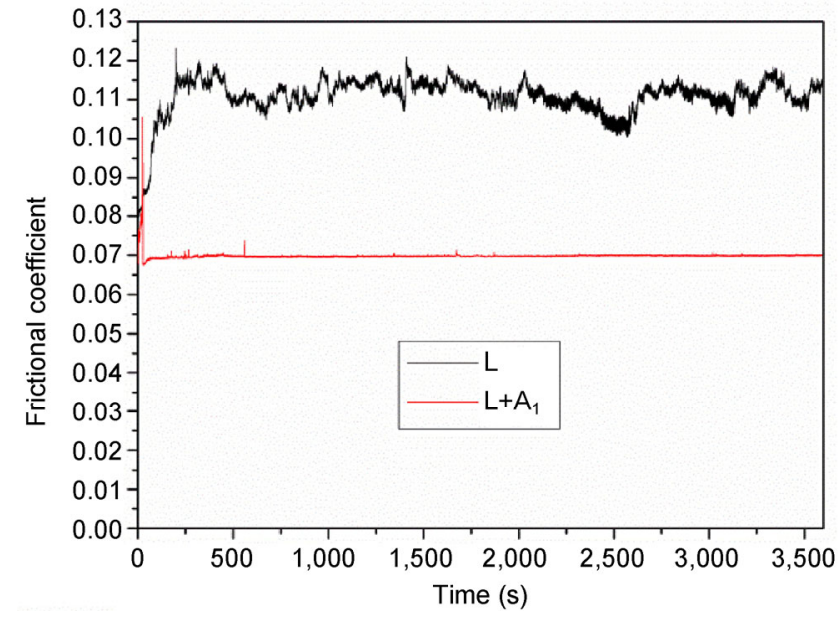

Fig. 7 Curves of frictional coefficients for pure 1,3,4-tri-(2octyldodecyl) cyclopentane and the mixture of tri-(2-octyldodecyl) phosphate with 1,3,4-tri-(2-octyldodecyl) cyclopentane.

that the tri-(2-octyldodecyl) phosphate is an excellent additive for 1,3,4-tri-(2-octyldodecyl) cyclopentane lubricant.

\section{Conclusions}

Tricresol phosphate is not suited as a lubricant additive for 1,3,4-tri-(2-octyldodecyl) cyclopentane lubricant due to its limited compatibility. Tri-(2-octyldodecyl) phosphate is an excellent lubricant additive due to perfect compatibility, ease of forming shear film on friction pairs, high film strength, and low energy dissipation. The mechanism of action is solid-liquid composite lubrication and the theoretical results were verified by experiment. In this study, a new testing 
method has been created for screening additives to lubricant oil.

\section{Acknowledgments}

This work is supported by the National Nature Science Foundation of China (Nos. 51227804, 51522510 and 51405477), the "Top Hundred Talents" Program of Chinese Academy of Sciences and the National Key Basic Research and Development (973) Program of China (No. 2013CB632300) for financial support. The authors thank Prof Jingcheng Hao, Shandong University, for providing access to the software.

\section{Appendix: Characterization of tris-(2-octyldodecyl) phosphate}

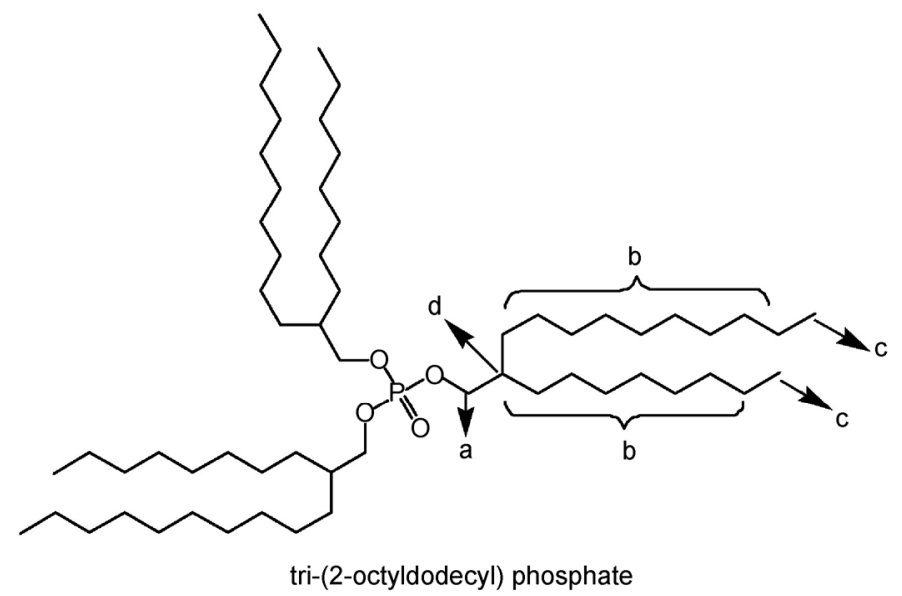

Fig. A1 The molecular structure of tri-(2-octyldodecyl) phosphate used for analyzing the IR and NMR spectra.

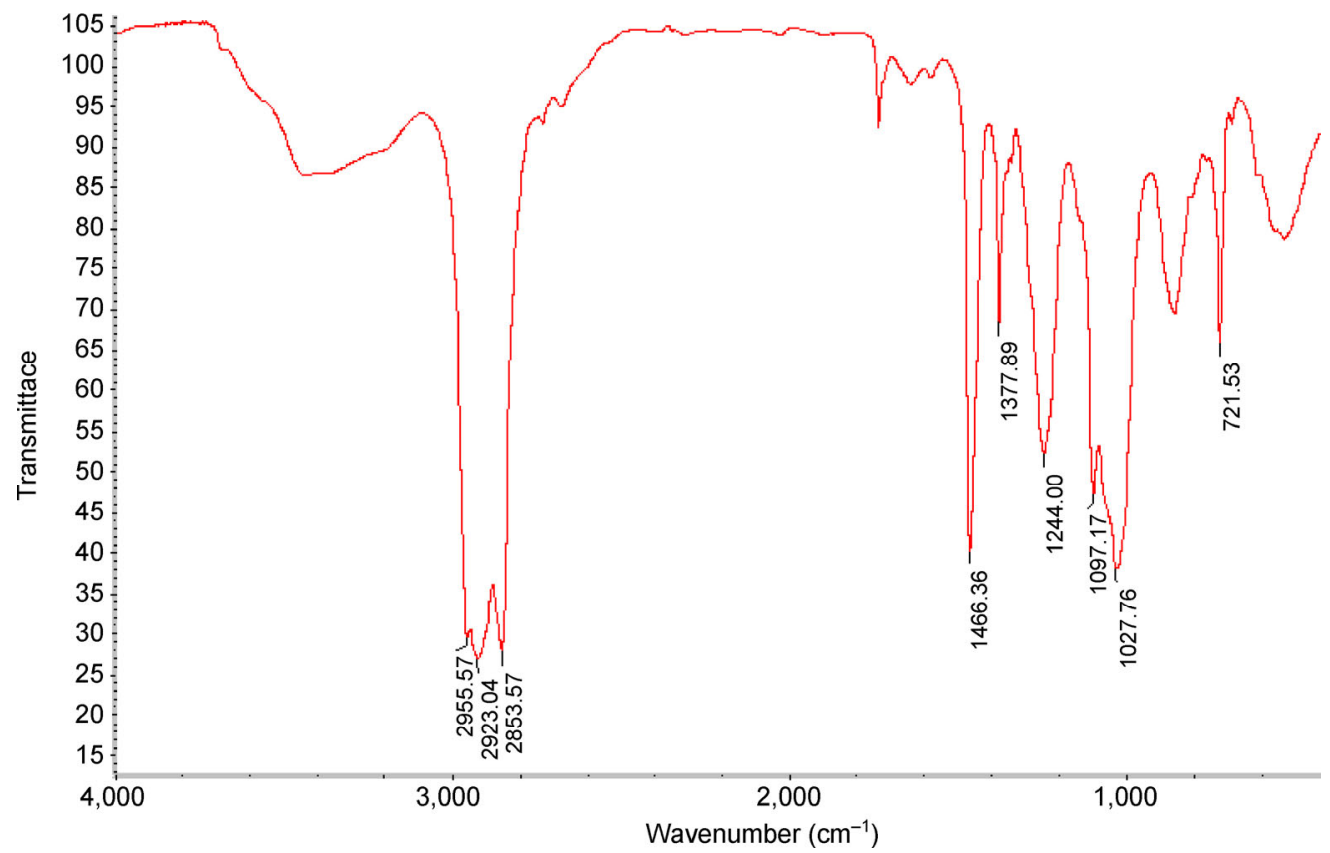

Fig. A2 The infrared spectrum of tri-(2-octyldodecyl) phosphate. IR (liquid film): $2955.57 \mathrm{~cm}^{-1}\left(-\mathrm{CH}_{3}\right), 2923.04 \mathrm{~cm}^{-1}\left(-\mathrm{CH}_{2}\right), 2853.57 \mathrm{~cm}^{-1}$ $\left(-\mathrm{CH}_{2}\right), 1466.36 \mathrm{~cm}^{-1}\left(-\mathrm{CH}_{3},-\mathrm{CH}_{2}\right), 1377.89 \mathrm{~cm}^{-1}\left(-\mathrm{CH}_{2}\right), 1244.00 \mathrm{~cm}^{-1}(-\mathrm{P}=\mathrm{O}), 1027.76 \mathrm{~cm}^{-1}(\mathrm{O}=\mathrm{P}-\mathrm{O}-\mathrm{C}), 721.53 \mathrm{~cm}^{-1}\left(-\left(\mathrm{CH}_{2}\right) x, x \geq 4\right)$. 


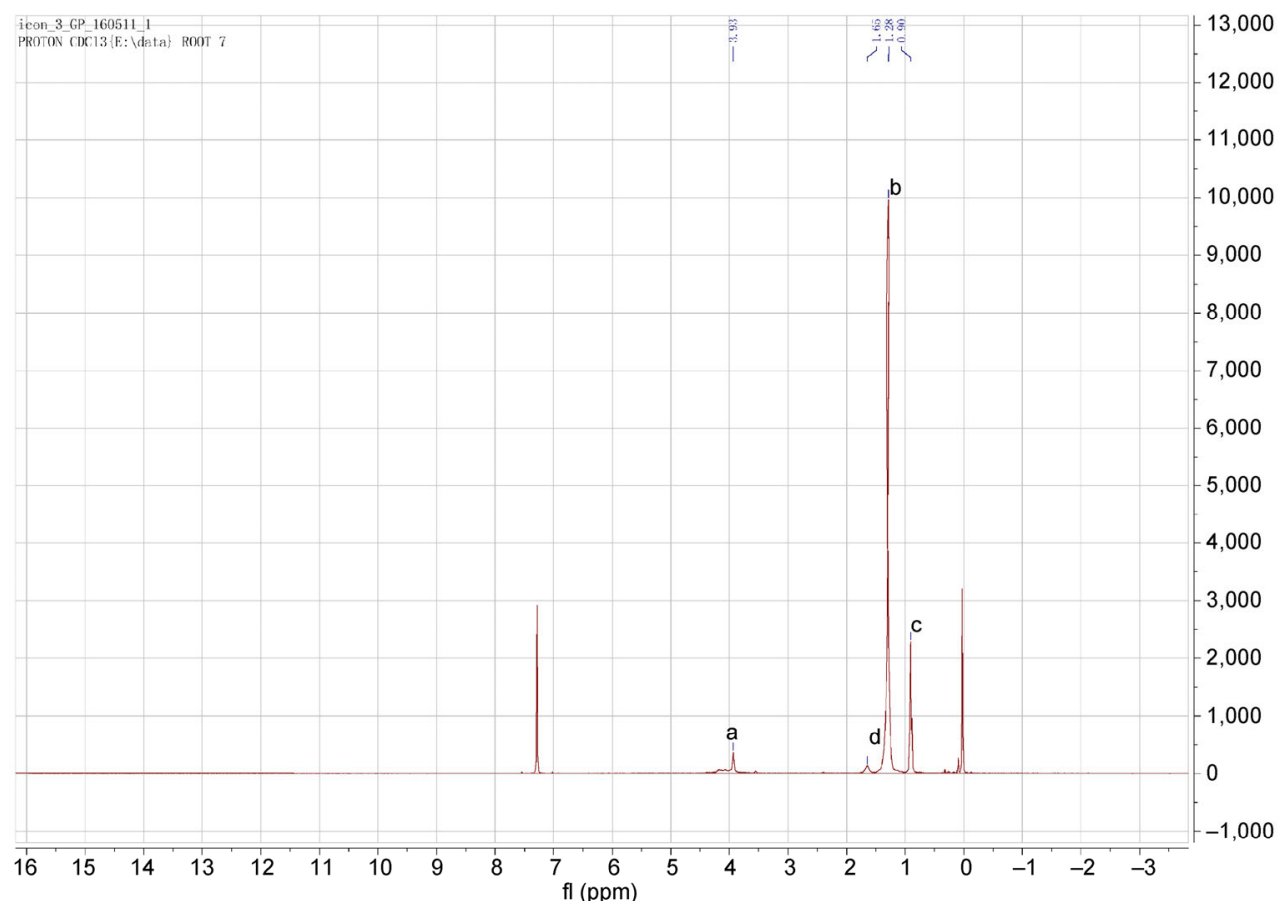

(a) ${ }^{1} \mathrm{H}: \delta-3.93(\mathrm{a}-6 \mathrm{H}), \delta-1.65(\mathrm{~d}-3 \mathrm{H}), \delta-1.28(\mathrm{~b}-32 \mathrm{H}), \delta-0.90(\mathrm{c}-18 \mathrm{H})$

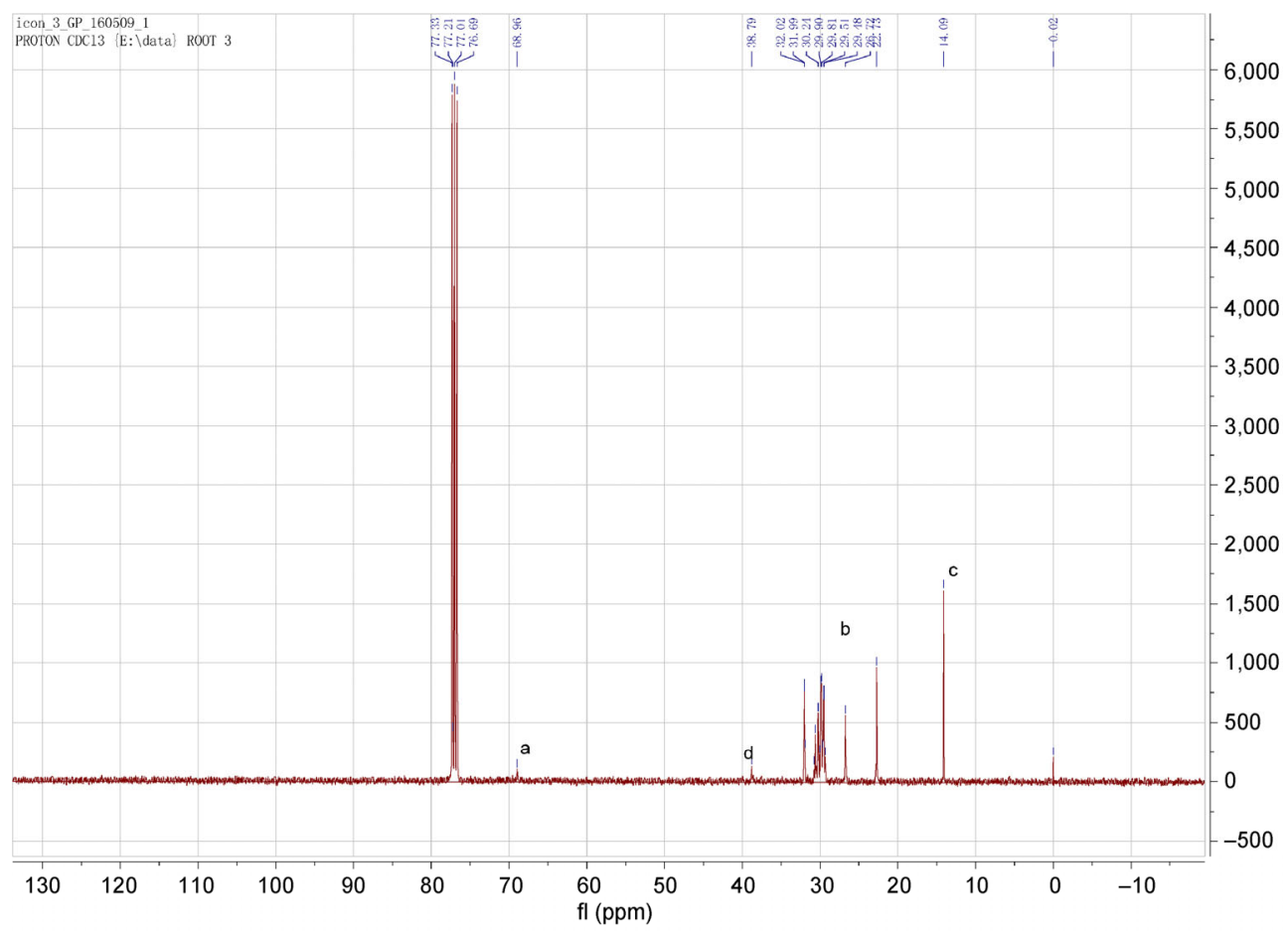

(b) ${ }^{13} \mathrm{C}: \delta-68.96$ (a), $\delta-38.79$ (d), $\delta-32.02 \sim 22.13$ (b), $\delta-14.09$ (c)

Fig. A3 NMR spectra of tri-(2-octyldodecyl) phosphate. 


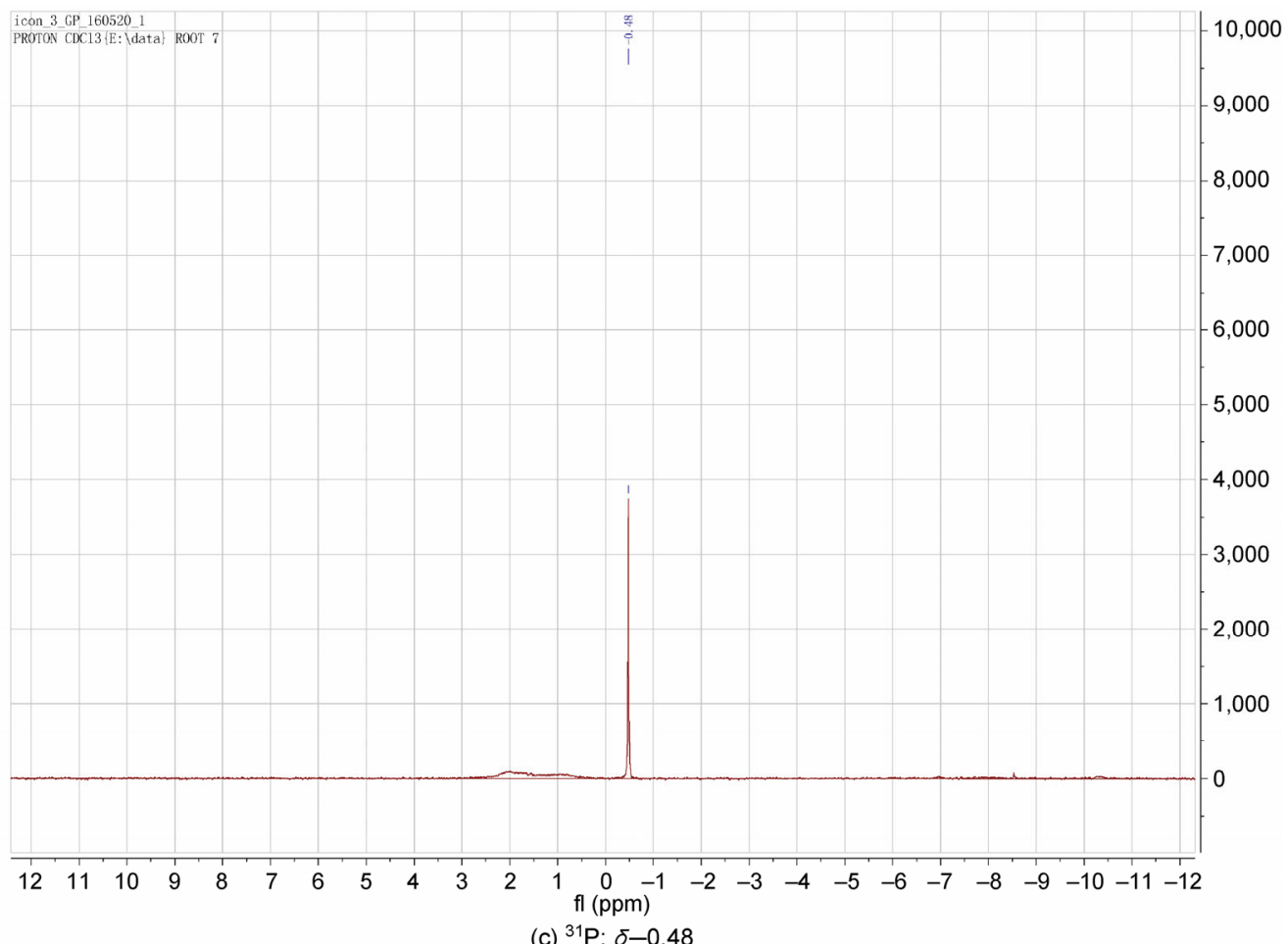

Fig. A3 (Continued)

Open Access: The articles published in this journal are distributed under the terms of the Creative Commons Attribution 4.0 International License (http:// creativecommons.org/licenses/by/4.0/), which permits unrestricted use, distribution, and reproduction in any medium, provided you give appropriate credit to the original author(s) and the source, provide a link to the Creative Commons license, and indicate if changes were made.

\section{References}

[1] Gschwender L J, Snyder C E J, Massey M, Peterangelo S C. Improved liquid/grease lubricants for space mechanisms. Lubr Eng 56(12): 25-31 (2000)

[2] Nian J Y, Gao P, Wang Y C, Guo Z G, .Liu W M. Theoretical investigation of atomic oxygen erosion mechanisms of 1,3-didecyl cyclopentane, 1,3-dioctyldodecyl cyclopentane and alkylated cyclopentane. RSC Adv 4: 50486-50493 (2014)

[3] Venier C G, Casserly E W. Multiply-alkylated cyclopentanes (MACs): A new class of synthesized hydrocarbon fluids. Lubr Eng 47: 586-591 (1991)

[4] Dube M J, Bollea D, Jones W R, Marrcheti M, Jansen M J. A new class of synthetic hydrocarbon fluid lubricantfor space applications. Tribol Lett 15: 3-8 (2003)
[5] Ma J Q, Pang C J, Mo Y F, Bai M W. Preparation and tribological properties of multiply-alkylated cyclopentane (MAC)-octadecyltrichlorosilane (OTS) double-layer film on silicon. Wear 263: 1000-1007 (2007)

[6] Sun C F, Zhang M, Zhou F, Gao P, Xia Y Q, Liu W M. tribological behavior of multiply-alkylated cyclopentanes (MACs)-Cu nanoparticles composite thin film. J Macromol Sci Part B: Phys 50: 1006-1017 (2011)

[7] Braza J, Jansen M J, Jones W. R. Lubricated bearing lifetimes of a multiply alkylated cyclopentane and a linear perfluoropolyether fluid in oscillatory motion at elevated temperatures in ultrahigh vacuum. NASA CR 21: 5637 (2009)

[8] Song Z H, Cai M R, Liang Y M, Fan M J, Zhou F, Liu W M. In situ preparation of anti-corrosion ionic liquids as the lubricant additives in multiply-alkylated cyclopentanes. RSC Adv 3: 21715-21721 (2013)

[9] Mo Y, Chau A, Wan Y, Lu Z, Huang F. Fabrication and tribological properties of a multiply-alkylated cyclopentane/ reduced graphene oxide composite ultrathin film. Carbon 65: 261-268 (2013)

[10] Fan X Q, Wang L P. Graphene with outstanding antiirradiation capacity as multialkylated cyclopentanes additive toward space application. Sci Rep 5: 12734 (2015)

[11] Biebera H E, Klausb E E, Tewksbury E J. A study of tricresyl phosphate as an additive for boundary lubrication. A S L E Trans 11: 155-161 (1968) 
[12] Peterangelo S C, Gschwender L, Jr C E S, Nguyen W R J Q G, Jansen M J. Improved additives for multiply alkylated cyclopentane-based lubricants. J Synth Lubr 25: 31-41(2008)

[13] Didziulis S V, Fleischauer P D. Chemistry of the extremepressure lubricant additive lead naphthenate on steel surfaces. Langmuir 12(7): 2981-2990 (1991)

[14] Bird R J, Galvin G D. The application of photoelectron spectroscopy to the study of EP films on lubricated surfaces. Wear 34: 143-167 (1976)

[15] Fan C F, Olafson B D, Blanco M. Hsu S L. Application of molecular simulation to derive phase diagrams of binary mixtures. Macromolecules 25: 3667 (1992)

[16] Pesci A I, Freed K F. Lattice models of polymer fluids: Monomers occupying several lattice sites. II. Interaction energies. J Chem Phys 90: 2003 (1989)

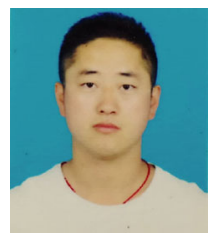

Jingyan NIAN. He received his master degree in physical chemistry in 2012 from Northwest Normal University, Lanzhou, China. During his undergraduate, he joined the State

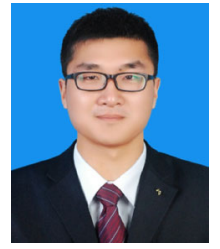

Yifan SI. He joined Prof. Guo's biomimetic materials of tribology (BMT) group at University of Hubei in 2014 in pursuing his Ph.D. degree.

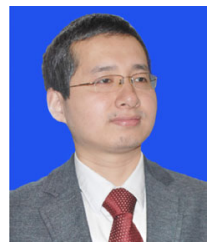

Zhiguang GUO. He received his Ph.D. degree from Lanzhou Institute of Chemical Physics (LICP), Chinese Academy of Sciences (CAS) in 2007. During Feb. 2009 to Feb. 2010, he

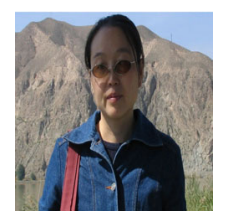

Ping GAO. She got her B.S. degree in 1989 in Lanzhou University, China. She is currently an associate professor

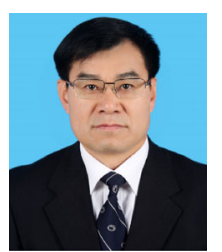

Weimin LIU. He received his Ph.D. degree with major of lubricating materials and tribology from Lanzhou Institute of Chemical Physics (LICP), Chinese Academy of Sciences in 1990. In 2013, he was elected as the fellow of
[17] Flory P J. Principles of Polymer Chemistry. Ithaca: Cornell University Press, 1953.

[18] Mayo S L, Olafson B D, Goddard W A. DREIDING: A generic force field for molecular simulations. J Phys Chem 94: 8897-8909(1990)

[19] Green M S. Markoff random processes and the statistical mechanics of time-dependent phenomena. II. Irreversible processes in fluids. J Chem Phys 22(3): 398 (1954)

[20] Wang J H. Synthesis of Triphosphate and Triphosphorous Acid Ester. Leather Chem 5: 26-29 (1998)

[21] Gao P, Liu W M, Liang Y M, Sun C F. Method for preparing Guerbet alcohol. CN Patent 102020533A, Apr. 2011.

[22] Venier C G, Casserly E W. Lubricants comprising novel cyclopentanes, cyclopentadienes, cyclopentenes, and mixtures thereof and methods of manufacture. US Patent 5012023, 1991.

Key Laboratory of Solid Lubrication at Lanzhou Institute of Chemical Physics, Chinese Academy of Sciences. His current position is an associate researcher. His research area covers the atomic-scale friction, superlubricity, and space lubrication.

His current scientific interests are devoted to designing and fabricating superhydrophobic nanocoatings and studying their corresponding applications.

worked in Department of Physics, University of Oxford, UK, as a visiting scholar. Now he is a full professor in LICP financed by "Top Hundred Talents" program of CAS. Till now, he has published more than 130 papers about the interfaces of materials.

in State Key Lab of Solid Lubrication, LICP, CAS. Her research interests mainly include organic synthesis and chemistry of materials.

Chinese Academy of Sciences, and now, he is the director of the state key lab of solid lubrication. Up to now, he has published more than 500 papers and his research interests mainly focus on space lubrication and high performance lubricants. 